\title{
Successful treatment of neonatal severe hyperparathyroidism with cinacalcet in two patients
}

\author{
Marisa M Fisher ${ }^{1}$, Susanne M Cabrera ${ }^{2}$ and Erik A Imel ${ }^{1,3}$ \\ ${ }^{1}$ Division of Pediatric Endocrinology, Department of Pediatrics, Riley Hospital for Children, Indiana University School \\ of Medicine, 705 Riley Hospital Drive, Room 5960, Indianapolis, Indiana 46220, USA \\ ${ }^{2}$ Division of Pediatric Endocrinology, Department of Pediatrics, Medical College of Wisconsin, Children's Hospital of \\ Wisconsin, 9000 W. Wisconsin Avenue, PO Box 1997, Milwaukee, Wisconsin 53201, USA \\ ${ }^{3}$ Division of Endocrinology, Department of Medicine, Indiana University School of Medicine, 541 North Clinical \\ Drive, Indianapolis, Indiana 46202, USA
}

\author{
Correspondence \\ should be addressed \\ to $\mathrm{M}$ M Fisher \\ Email \\ mamfishe@iu.edu
}

\section{Summary}

Neonatal severe hyperparathyroidism (NSHPT) is a rare disorder caused by inactivating calcium-sensing receptor (CASR) mutations that result in life-threatening hypercalcemia and metabolic bone disease. Until recently, therapy has been surgical parathyroidectomy. Three previous case reports have shown successful medical management of NSHPT with cinacalcet. Here we present the detailed description of two unrelated patients with NSHPT due to heterozygous R185Q CASR mutations. Patient 1 was diagnosed at 11 months of age and had developmental delays, dysphagia, bell-shaped chest, and periosteal bone reactions. Patient 2 was diagnosed at 1 month of age and had failure to thrive, osteopenia, and multiple rib fractures. Cinacalcet was initiated at 13 months of age in patient 1, and at 4 months of age in patient 2 . We have successfully normalized their parathyroid hormone and alkaline phosphatase levels. Despite the continuance of mild hypercalcemia (11-12 mg/dl), both patients showed no hypercalcemic symptoms. Importantly, patient 1 had improved neurodevelopment and patient 2 never experienced any developmental delays after starting cinacalcet. Neither experienced fractures after starting cinacalcet. Both have been successfully managed long-term without any significant adverse events. These cases expand the current literature of cinacalcet use in NSHPT to five successful reported cases. We propose that cinacalcet may be considered as an option for treating the severe hypercalcemia and metabolic bone disease found in infants and children with inactivating CASR disorders.

\section{Learning points:}

- NSHPT due to mutations in the CASR gene occurs with hypercalcemia and metabolic bone disease, but not always with severe critical illness in infancy.

- NSHPT should be considered in the differential diagnosis for a newborn with a bell-shaped chest, osteopenia, and periosteal reactions.

- Neurodevelopmental consequences may occur in children with hypercalcemia and may improve during treatment.

- Calcimimetics can be used to successfully treat the pathophysiology of NSHPT directly to control serum calcium levels. 


\section{Background}

The calcium-sensing receptor (CASR) is a 1078 amino acid cell surface protein with a large extracellular domain that tightly regulates calcium homeostasis (1). Under normal conditions, increasing extracellular calcium concentrations activate the CASR, diminishing parathyroid hormone (PTH) release and augmenting renal calcium excretion (2). Inactivating CASR gene mutations lead to variable degrees of hypercalcemia. Heterozygous mutations usually result in mild hypercalcemia seen in familial hypocalciuric hypercalcemia (FHH). Homozygous CASR mutations result in neonatal severe hyperparathyroidism (NSHPT), producing life-threatening hypercalcemia and extreme PTH and alkaline phosphatase elevations in the neonatal period. Patients usually present in the first weeks of life with failure to thrive, hypotonia, respiratory distress, and metabolic bone disease. Bone abnormalities can include undermineralization, rib cage deformities, subperiosteal erosions, metaphyseal widening of the long bones, and fractures (1).

Heterozygous CASR gene mutations resulting in the NSHPT phenotype have also been described (3) (4) (5). The heterozygous mutation, $\mathrm{R} 185 \mathrm{Q}$, is one such mutation and in vitro studies have demonstrated the dominant negative inhibition of the WT CASR by this mutant CASR (4) (6). Until recently, the only effective therapy for NSHPT was surgical parathyroidectomy (7). Despite improved surgical outcomes, this is still a complex procedure in very young patients (2) (8). Few surgeons have parathyroidectomy experience in infants due to the rarity of the disorder. The need for medical management of other PTH-dependent hypercalcemic disorders drove the development of allosteric CASR agonists, namely cinacalcet (9). Three previous case studies have shown the successful use of cinacalcet, in NSHPT (8) (10) (11). Here we report the cases of two unrelated patients with de novo R185Q heterozygous inactivating CASR mutations and highlight their rapid biochemical and clinical response to treatment with cinacalcet.

\section{Case presentation: case 1}

Patient 1 was a term male infant with global hypotonia, bell-shaped chest, and metaphyseal irregularities (Fig. 1) that led to an initial clinical diagnosis of Juene's syndrome. This is an autosomal recessive skeletal ciliopathy causing a short-rib thoracic dysplasia, short-limbed short stature, and severe thoracic constriction (12). The Juene's syndrome phenotype is variable but to our
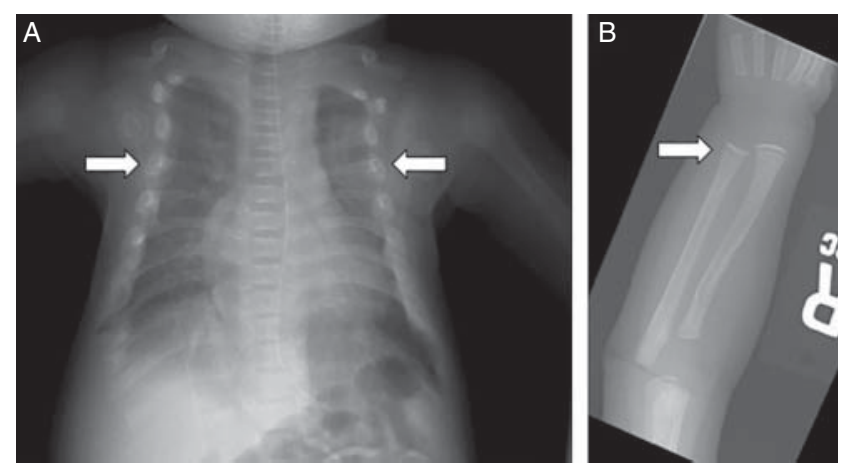

\section{Figure 1}

(A) Chest X-ray for patient 1, at 1 month of age, shows an elongated and narrowed rib cage with irregular rib ends (arrows). The rib cage was also narrow in an anteroposterior direction (not shown). (B) There are areas of sclerosis in the distal radius and ulna at 1 month of age (arrow).

knowledge does not include hypercalcemia (12). He later presented to endocrine care at age 11 months after additional review of the radiographs indicated diffuse osteopenia, short ribs with irregular rib ends, and metaphyseal sclerosis at the ends of multiple long bones, suggesting a metabolic bone disease, and biochemical evaluation revealed hypercalcemia (Table 1).

\section{Investigation: case 1}

Primary hyperparathyroidism was evident with hypercalciuria, consistent with excessive calcium load filtered at the glomerulus. Retrospectively, hypercalcemia $(11.7-12.9 \mathrm{mg} / \mathrm{dl})$ had been present on lab testing in the neonatal period, but had not been investigated until seen by endocrinology at 11 months of age. At this age he had gross motor, fine motor and speech delays and dysphagia requiring gastrostomy tube feedings. There was no family history of hypercalcemia or bone disease. Both parents were normocalcemic. Sequencing of the CASR gene revealed a heterozygous $\mathrm{R} 185 \mathrm{Q}$ missense mutation.

Initial therapy included $0.9 \%$ saline, phosphate supplementation, and a low-calcium formula. He had a transient response to a single dose of pamidronate $(0.5 \mathrm{mg} / \mathrm{kg}$ i.v. $)$, but serum calcium rose to $13.8 \mathrm{mg} / \mathrm{dl}$ after only 2 weeks. Therefore, definitive therapy was sought.

\section{Treatment: case 1}

Cinacalcet, $15 \mathrm{mg}$ orally twice daily $(3.7 \mathrm{mg} / \mathrm{kg}$ per day), was begun at 13 months of age based on dosing from the first published case report (11). The tablets were crushed 
Table 1 Patient biochemistries at NSHPT diagnosis.

\section{Lab}

Calcium

Albumin

Alkaline phosphatase

Phosphorus

Parathyroid hormone

Urine $\mathrm{Ca} / \mathrm{Cr}$

Vitamin D 25

Vitamin D 1,25

Magnesium
Patient 1 values at diagnosis at 11 months

$13.8 \mathrm{mg} / \mathrm{dl}$
$3.9 \mathrm{~g} / \mathrm{dl}$
$235 \mathrm{U} / \mathrm{l}$
$4.4 \mathrm{mg} / \mathrm{dl}$
$76 \mathrm{pg} / \mathrm{ml}$
$2.3 \mathrm{mg} / \mathrm{mg}$

$17 \mathrm{ng} / \mathrm{ml}$

$40 \mathrm{pg} / \mathrm{ml}$

$3.1 \mathrm{mg} / \mathrm{dl}$
Patient 2 values at diagnosis at 1 month

$13.3 \mathrm{mg} / \mathrm{dl}$

$3.1 \mathrm{~g} / \mathrm{dl}$

$372 \mathrm{U} / \mathrm{l}$

$4.4 \mathrm{mg} / \mathrm{dl}$

$196 \mathrm{pg} / \mathrm{ml}$

$<0.08 \mathrm{mg} / \mathrm{mg}$

$30.2 \mathrm{ng} / \mathrm{ml}$

$230 \mathrm{pg} / \mathrm{ml}$

$2.4 \mathrm{mg} / \mathrm{dl}$
Normal range for age

$8.5-10.5$

$3.1-4.2$

$88-351$

4.8-8.1

$10-65$

$<0.6$ (patient 1)

$<0.8$ (patient 2)

25-80

24-86

$1.6-2.9$ and dissolved in water for enteral administration. Calcium levels normalized $(10.4 \mathrm{mg} / \mathrm{dl})$ within 4 weeks and phosphorus levels rose to near normal $(4.3 \mathrm{mg} / \mathrm{dl})$ (Fig. 2A). The low-calcium formula was discontinued and calcium restriction lifted. The cinacalcet dose ranged from 30 to $60 \mathrm{mg} / \mathrm{day}(2.4-7.4 \mathrm{mg} / \mathrm{kg}$ per day) in two to four divided doses to target serum calcium levels below $12 \mathrm{mg} / \mathrm{dl}$ (Fig. 2B). Phosphorus supplementation was discontinued at 16 months of age. PTH and alkaline phosphatase levels normalized with only brief elevations and ranged between $30-78 \mathrm{pg} / \mathrm{ml}$ and $209-565 \mathrm{U} / 1$ respectively.
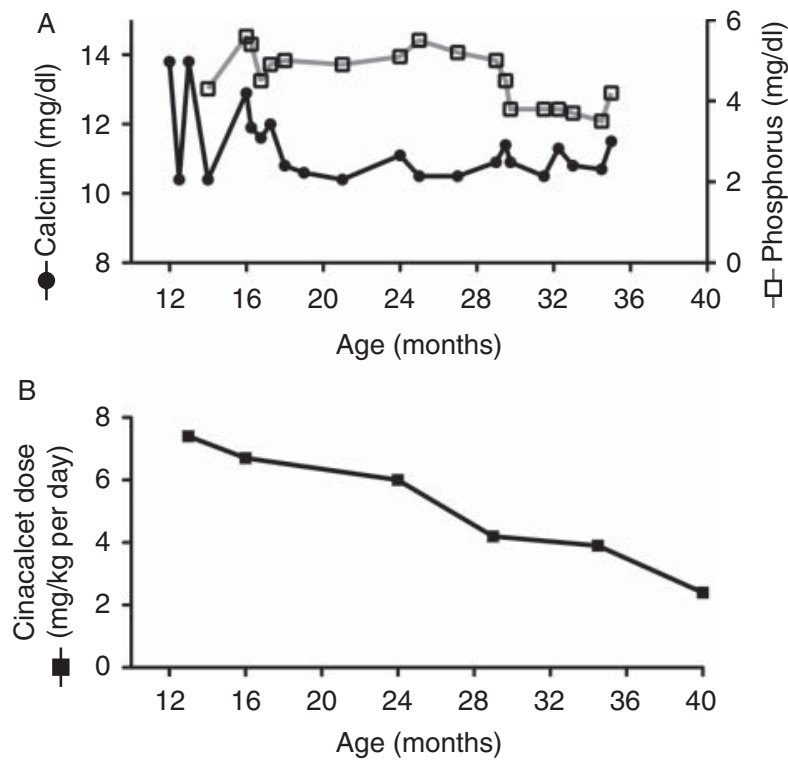

Figure 2

(A) Longitudinal calcium (black circles) and phosphorus (open squares) values during cinacalcet treatment and $(B)$ corresponding cinacalcet dosing ( $\mathrm{mg} / \mathrm{kg}$ per day). The $\mathrm{mg} / \mathrm{kg}$ per day dosing is approximate, as dose changes were made more frequently than weight checks.

\section{Outcome and follow-up: case 1}

To date, patient 1 has continued cinacalcet monotherapy for 32 months without adverse incident. Calcium levels have remained slightly elevated (10.4-12 mg/dl) except for one time point when a dose increase was required. PTH levels have been maintained in the normal range. Our patient experienced mild nausea and vomiting after one dose increase, but these well-known side effects of cinacalcet resolved with a dose decrease. His developmental delays improved slowly and at 3 years of age he no longer required interventional services. His dysphagia resolved and his gastrostomy tube was removed 5 months after starting cinacalcet. His weight (3-10th percentile) and height (10-25th percentile) steadily increased while taking cinacalcet. He has not experienced fractures and does not complain of bone pain.

\section{Case presentation: case 2}

Patient 2, a term female infant, presented at 26 days of age with failure of linear growth, poor weight gain, and cough. A chest X-ray was obtained showing multiple rib fractures. Subsequently, a skeletal survey revealed diffusely osteopenic bones with coarse trabecular markings, diffuse symmetric periosteal reactions, and healing lateral right fourth to ninth and left seventh to ninth rib fractures (Fig. 3).

\section{Investigation: case 2}

Laboratory evaluation revealed hyperparathyroidism (Table 1). There was no family history of hypercalcemia or bone disease. Both parents were normocalcemic. Sequencing of the CASR gene revealed a heterozygous R185Q missense mutation. Infusion of fluids with $0.9 \%$ saline, oral phosphorus supplementation, and a 


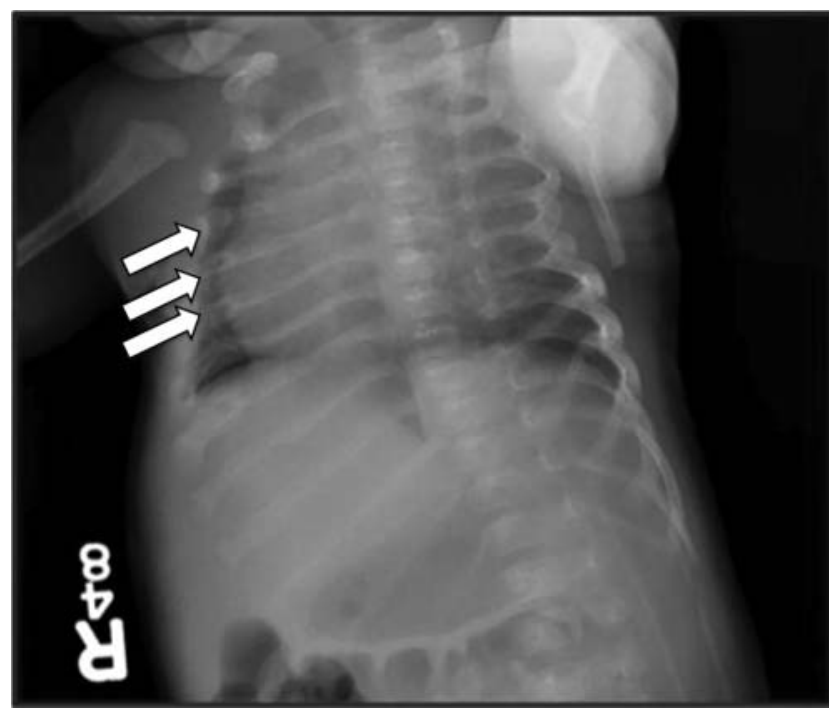

Figure 3

Chest X-ray for patient 2, at 4 weeks of age, shows generalized osteopenia and numerous multilevel bony callus formations along several contiguous ribs bilaterally, suggesting healing fractures (arrows) involving ribs two to nine on the right and ribs two to seven on the left.

low-calcium formula were initiated. Calcium levels decreased to 11-12 $\mathrm{mg} / \mathrm{dl}$, but the patient had worsening respiratory distress and was diagnosed with adenovirus. She required ventilator support for 5 days. Notably, calcium levels were controlled during this time (11.4-11.5 mg/dl). Her respiratory distress resolved and after demonstrating adequate weight gain she was discharged on only a low-calcium formula and phosphorus supplementation. She maintained a calcium level generally below $12 \mathrm{mg} / \mathrm{dl}$ but significant PTH and alkaline phosphatase elevations developed over the next 12 weeks (Fig. $4 \mathrm{~A}$ and B).

\section{Treatment: case 2}

Cinacalcet, $6 \mathrm{mg}$ orally daily ( $2 \mathrm{mg} / \mathrm{kg}$ per day), was initiated at 4 months of age. Our hospital's pharmacy made a compounded solution $(6 \mathrm{mg} / \mathrm{ml})$ to allow for small doses. Initial dosing and titration was done in an inpatient environment to monitor for side effects and obtain frequent laboratory monitoring. A decrease in her calcium level from 12.5 to $11.9 \mathrm{mg} / \mathrm{dl}$ was noted over $18 \mathrm{~h}$. Cinacalcet dose titrations normalized her PTH in 11 days and her serum calcium remained within our goal of $<12 \mathrm{mg} /$ dl during this time (Fig. $4 \mathrm{~A}, \mathrm{~B}$ and C). Alkaline phosphatase decreased gradually and normalized after 15 weeks of cinacalcet therapy. Our patient remained mildly hypercalcemic (10.5-12.7 mg/dl) and dose increases were performed for values above $12 \mathrm{mg} / \mathrm{dl}$ while targeting a normal PTH and alkaline phosphatase. She experienced no symptoms from the intermittent hypercalcemia, and the highest calcium values were noted after missing cinacalcet doses. Specific doses ranged between $6 \mathrm{mg}$ daily and $10 \mathrm{mg}$ three times a day orally $(1.68-2.7 \mathrm{mg} / \mathrm{kg}$ per day) over 13 months of therapy.

\section{Outcome and follow-up: case 2}

She experienced no adverse side effects from cinacalcet, including hypocalcemia. A renal ultrasound at 1 month of age (before therapy) demonstrated mild nephrocalcinosis
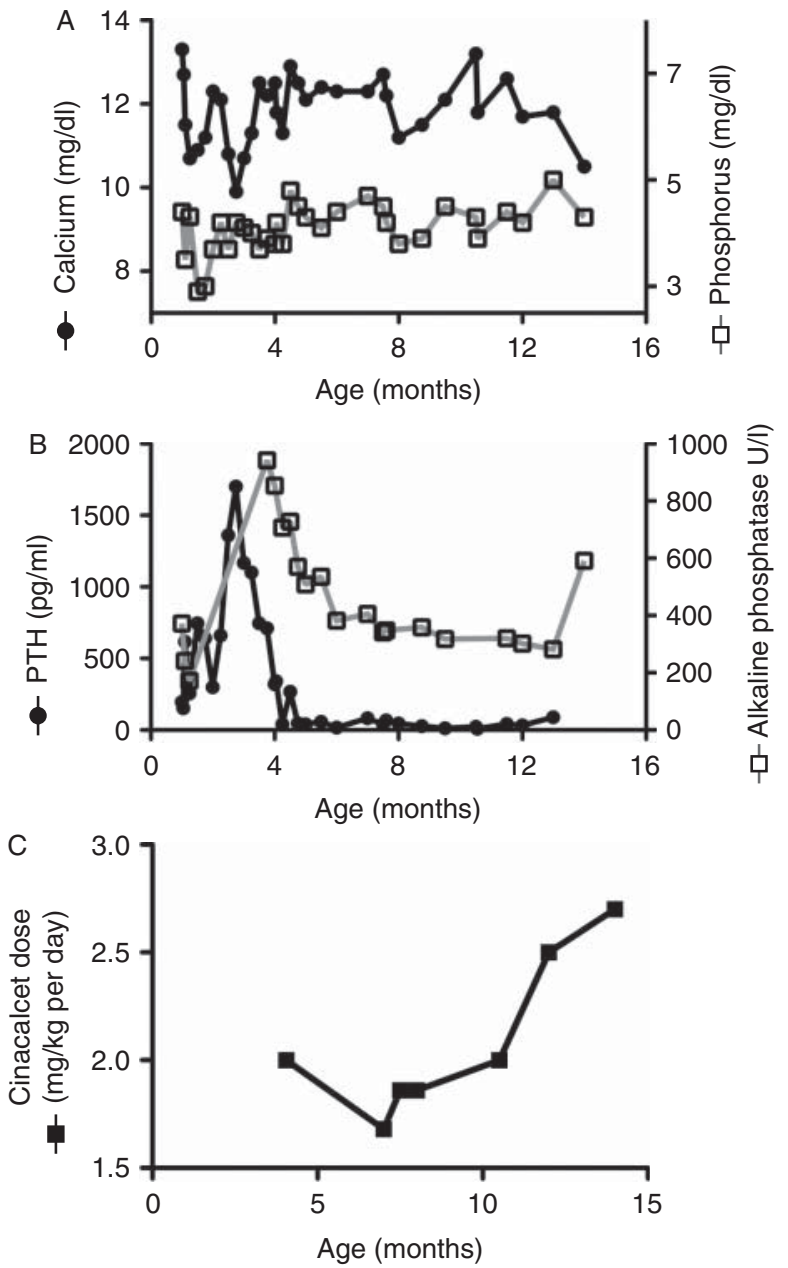

\section{Figure 4}

(A) Longitudinal calcium (black circles) and phosphorus (open squares), (B) PTH (black circles) and alkaline phosphatase (open squares) values before and during cinacalcet treatment, and (C) corresponding cinacalcet dosing ( $\mathrm{mg} / \mathrm{kg}$ per day). The $\mathrm{mg} / \mathrm{kg}$ per day dosing is approximate as dose changes were made more frequently than weight checks. 
that was stable at 9 months of age. Her rib fractures demonstrated healing by radiograph at 4 months of age and she experienced no further fractures. She had improved weight gain after reduction in her calcium levels ( $\sim 50$ th percentile for weight and $\sim 25$ th percentile for length at 4 months), which continued after starting cinacalcet $(\sim 90$ th percentile for weight and $\sim 75$ th percentile for length at 12 months). Her development throughout her first year of life was normal and without any noted delays.

\section{Discussion}

We present the successful medical management of symptomatic hypercalcemia due to NSHPT with cinacalcet mono-therapy. Our cases responded similarly to the three reported cases of NSHPT treated successfully with cinacalcet and reinforce that medical management without surgery can successfully control the hypercalcemia and its sequelae in NSHPT due to the dominant negative heterozygous $\mathrm{R} 185 \mathrm{Q}$ mutation. The rare nature of NSHPT makes it unlikely that randomized clinical trials will be feasible, highlighting the value of detailed case reports.

To date, there have been three published cases of NSHPT successfully treated with cinacalcet (reviewed in Table 2) (8) (10) (11). The first, in 2011, was an infant with the heterozygous $\mathrm{R} 185 \mathrm{Q}$ CASR mutation treated with cinacalcet at 3 weeks of age (11). This patient responded well with long-term normalization of PTH levels and near normalization of calcium levels without reported side effects (11). The second report, in 2012, described a child with a homozygous CASR mutation treated with cinacalcet at 6 years of age with normalization of PTH and near normalization of calcium levels (8). The third case, in 2014, was a newborn with the heterozygous R185Q mutation (10). This patient had a similar course and the authors emphasized that calcium levels may not normalize because of the dominant inhibitor effect of the abnormal CASR protein (10).

Both of our patients also had continued mild hypercalcemia with normalization of PTH and phosphorus levels despite increased cinacalcet doses. We targeted a calcium level below $12 \mathrm{mg} / \mathrm{dl}$ for several reasons. Levels in this range are less likely to induce symptoms and patients with $\mathrm{FHH}$ have similar levels without adverse long-term effects.

Our first patient had a bell-shaped chest, a feature that has been observed in other case reports of patients with a R185Q CASR mutation (10) (13). He initially received a
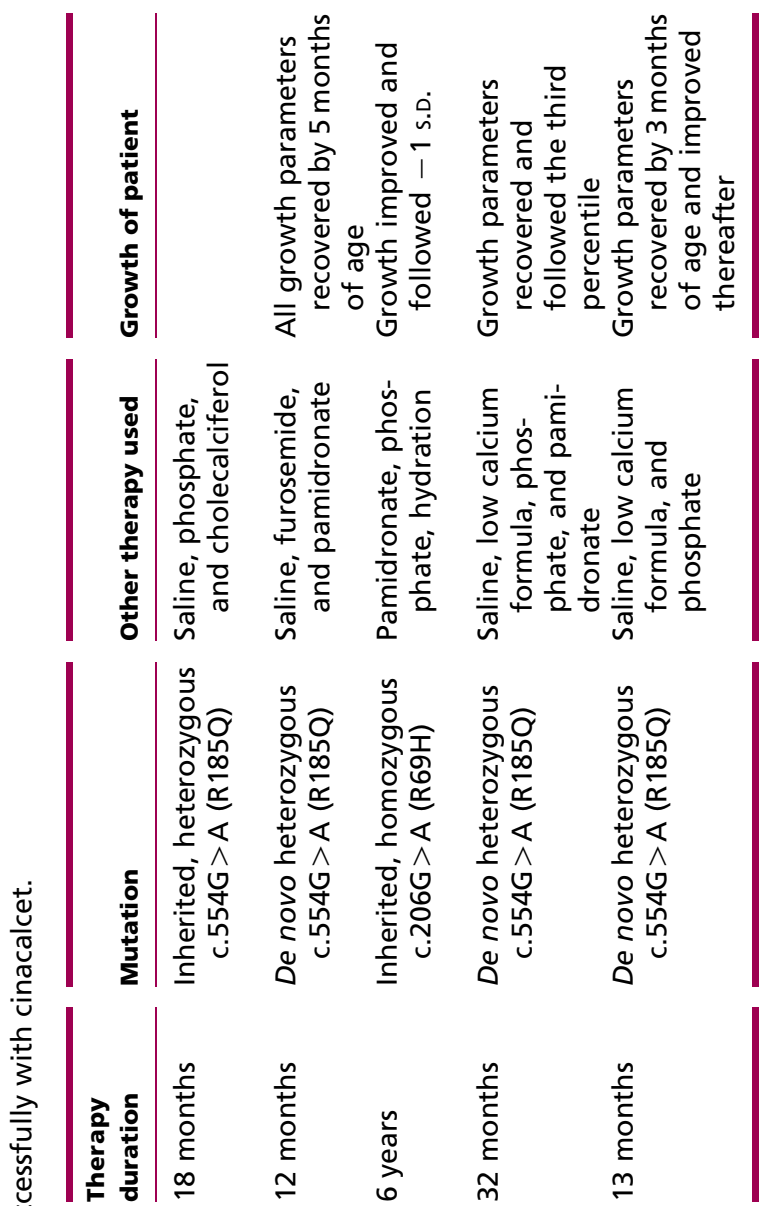

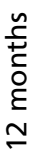

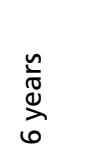

$\stackrel{n}{c}$
$+\frac{c}{c}$
ह

$\stackrel{n}{c}$
$\stackrel{+}{c}$
ह
$m$


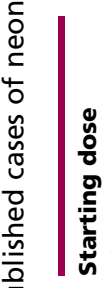
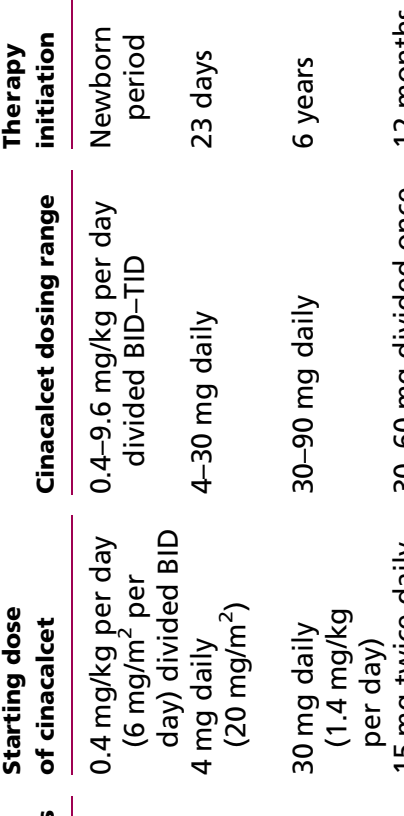



iे

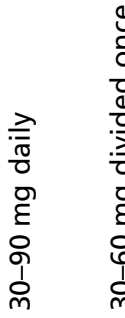

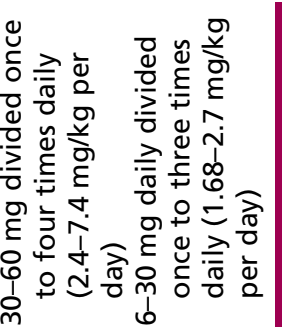

\begin{tabular}{l|l}
0 \\
$\frac{0}{0}$
\end{tabular}
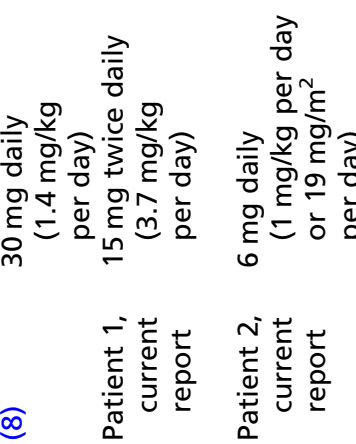
diagnosis of Juene's syndrome, leading to a delay in NSHPT diagnosis. Therefore, NSHPT should be in the differential of a newborn with a bell-shaped chest, osteopenia, and periosteal reactions.

Hypercalcemia can cause many devastating symptoms, including the developmental delays seen in our first patient. Importantly, he had marked improvement once his calcium levels decreased. Our second patient was treated at a young age and hence did not experience developmental delays. Both patients gained weight and height well and experienced no significant adverse side effects directly from cinacalcet therapy.

Cinacalcet was not initiated immediately at diagnosis in patient 2 for several reasons: she had initial clinical and biochemical improvement with low-calcium formula alone; there were reports that hyperparathyroidism in an infant with a heterozygous inactivating CASR mutation born to a normocalcemic mother may improve over time (11) (14); and a clinical trial investigating cinacalcet use for hyperparathyroidism in pediatric patients with chronic kidney disease had just announced a death and been halted (15). However, evidence of worsening skeletal turnover necessitated beginning treatment and after discussion with the parents regarding the risks and benefits of the alternatives, cinacalcet was chosen and consent for treatment signed. Although no long-term pediatric safety analysis is available for cinacalcet, we propose continued monitoring of calcium, phosphorus, PTH, and alkaline phosphatase levels to direct dosing and ongoing intermittent monitoring of kidney function, urinary calcium, and renal ultrasounds. We plan to continue therapy indefinitely, as the alternative requires parathyroid surgery to manage serum calcium. It may, however, be possible to taper off treatment while monitoring serum calcium, as our first patient has had a steady decrease in his dose as he has grown (Fig. 2B).

Despite the above reported successes, in vitro studies have shown varying effects of cinacalcet in different CASR mutations, suggesting that cinacalcet may not be effective in every case. The residual functionality of the CASR protein and location of the mutation in the transmembrane receptor may be important predictors of success with cinacalcet treatment (16). In accordance, two cases of NSHPT have been described where cinacalcet therapy failed (13) (17). These cases had different CASR mutations from each other, but both resulted in a nonfunctional protein due to truncating mutations. A cataloguing of CASR mutations and their response to therapy may be a valuable asset in determining treatment for NSHPT.
In conclusion, we report two cases of NSHPT caused by a heterozygous inactivating dominant negative CASR mutation that responded very well to cinacalcet monotherapy. Our patients also showed improvements in bone structure and did not fracture while on cinacalcet therapy. While the response to cinacalcet will be dictated by residual function of the CASR protein, we suggest that a trial of cinacalcet be undertaken in infants and children with significant hypercalcemia and metabolic bone disease secondary to CASR mutations.

Declaration of interest

The authors declare that there is no conflict of interest that could be perceived as prejudicing the impartiality of the research reported.

\section{Funding}

This publication was supported by the NIDDK of the National Institutes of Health (award numbers T32DK065549 (M M Fisher) and K23AR057096 (E A Imel)).

\section{Patient consent}

Written informed consent has been obtained from the guardians for both patients for the publication of this submitted article and accompanying images. The journal's signed informed consent forms are included with this submission.

\section{Author contribution statement}

M M Fisher provided care for patient 2, researched literature for this manuscript and wrote the manuscript. S M Cabrera provided care for patient 1, wrote and edited this manuscript. E A Imel provided care for patient 1 , assistance with care for patient 2 , researched literature for this manuscript, wrote and edited this manuscript.

\section{References}

1 Gunn IR \& Gaffney D 2004 Clinical and laboratory features of calciumsensing receptor disorders: a systematic review. Annals of Clinical Biochemistry 41 441-458. (doi:10.1258/0004563042466802)

2 Roizen J \& Levine MA 2012 Primary hyperparathyroidism in children and adolescents. Journal of the Chinese Medical Association 75 425-434. (doi:10.1016/j.jcma.2012.06.012)

3 Hannan FM, Nesbit MA, Zhang C, Cranston T, Curley AJ, Harding B, Fratter C, Rust N, Christie PT, Turner JJ et al. 2012 Identification of 70 calcium-sensing receptor mutations in hyper- and hypo-calcaemic patients: evidence for clustering of extracellular domain mutations at calcium-binding sites. Human Molecular Genetics 21 2768-2778. (doi:10.1093/hmg/dds105)

4 Obermannova B, Banghova K, Sumnik Z, Dvorakova HM, Betka J, Fencl F, Kolouskova S, Cinek O \& Lebl J 2009 Unusually severe phenotype of neonatal primary hyperparathyroidism due to a heterozygous inactivating mutation in the CASR gene. European Journal of Pediatrics 168 569-573. (doi:10.1007/s00431-008-0794-y)

5 Pearce SH, Trump D, Wooding C, Besser GM, Chew SL, Grant DB, Heath DA, Hughes IA, Paterson CR, Whyte MP et al. 1995 Calcium-sensing 
Endocrinology

Diabetes \& Metabolism

CASE REPORTS
M M Fisher and others
Course of cinacalcet treatment in NSHPT
ID: 15-0040; July 2015 DOI: 10.1530/EDM-15-0040 receptor mutations in familial benign hypercalcemia and neonatal hyperparathyroidism. Journal of Clinical Investigation 96 2683-2692. (doi:10.1172/JCI118335)

6 Bai M, Pearce SH, Kifor O, Trivedi S, Stauffer UG, Thakker RV, Brown EM \& Steinmann B 1997 In vivo and in vitro characterization of neonatal hyperparathyroidism resulting from a de novo, heterozygous mutation in the $\mathrm{Ca}^{2+}$-sensing receptor gene: normal maternal calcium homeostasis as a cause of secondary hyperparathyroidism in familial benign hypocalciuric hypercalcemia. Journal of Clinical Investigation 99 88-96. (doi:10.1172/JCI119137)

7 Waller S, Kurzawinski T, Spitz L, Thakker R, Cranston T, Pearce S, Cheetham T \& van't Hoff WG 2004 Neonatal severe hyperparathyroidism: genotype/phenotype correlation and the use of pamidronate as rescue therapy. European Journal of Pediatrics 163 589-594. (doi:10.1007/s00431-004-1491-0)

8 Wilhelm-Bals A, Parvex P, Magdelaine C \& Girardin E 2012 Successful use of bisphosphonate and calcimimetic in neonatal severe primary hyperparathyroidism. Pediatrics 129 e812-e816. (doi:10.1542/peds. 2011-0128)

9 Filopanti M, Corbetta S, Barbieri AM \& Spada A 2013 Pharmacology of the calcium sensing receptor. Clinical Cases in Mineral and Bone Metabolism 10 162-165.

10 Gannon AW, Monk HM \& Levine MA 2014 Cinacalcet monotherapy in neonatal severe hyperparathyroidism: a case study and review. Journal of Clinical Endocrinology and Metabolism 99 7-11. (doi:10.1210/jc.2013-2834)

11 Reh CM, Hendy GN, Cole DE \& Jeandron DD 2011 Neonatal hyperparathyroidism with a heterozygous calcium-sensing receptor (CASR) R185Q mutation: clinical benefit from cinacalcet. Journal of
Clinical Endocrinology and Metabolism 96 E707-E712. (doi:10.1210/ jc.2010-1306)

12 Huber C \& Cormier-Daire V 2012 Ciliary disorder of the skeleton. American Journal of Medical Genetics. Part C, Seminars in Medical Genetics 160C 165-174. (doi:10.1002/ajmg.c.31336)

13 Atay Z, Bereket A, Haliloglu B, Abali S, Ozdogan T, Altuncu E, Canaff L, Vilaca T, Wong BY, Cole DE et al. 2014 Novel homozygous inactivating mutation of the calcium-sensing receptor gene (CASR) in neonatal severe hyperparathyroidism-lack of effect of cinacalcet. Bone 64 102-107. (doi:10.1016/j.bone.2014.04.010)

14 Pearce S \& Steinmann B 1999 Casting new light on the clinical spectrum of neonatal severe hyperparathyroidism. Clinical Endocrinology 50 691-693. (doi:10.1046/j.1365-2265.1999. 00788.x)

15 U.S. Food and Drug Administration. Sensipar (cinacalcet hydrochloride): drug safety communication - FDA suspends pediatric clinical trials after report of death, 2013. (http://www.fda.gov/Drugs/DrugSafety/ ucm340551.htm)

16 Zhang Z, Qiu W, Quinn SJ, Conigrave AD, Brown EM \& Bai M 2002 Three adjacent serines in the extracellular domains of the CaR are required for L-amino acid-mediated potentiation of receptor function. Journal of Biological Chemistry 277 33727-33735. (doi:10.1074/jbc. M200976200)

17 Garcia Soblechero E, Ferrer Castillo MT, Jimenez Crespo B, Dominguez Quintero ML \& Gonzalez Fuentes C 2013 Neonatal hypercalcemia due to a homozygous mutation in the calcium-sensing receptor: failure of cinacalcet. Neonatology 104 104-108. (doi:10.1159/000350540)

Received in final form 18 May 2015

Accepted 18 June 2015 